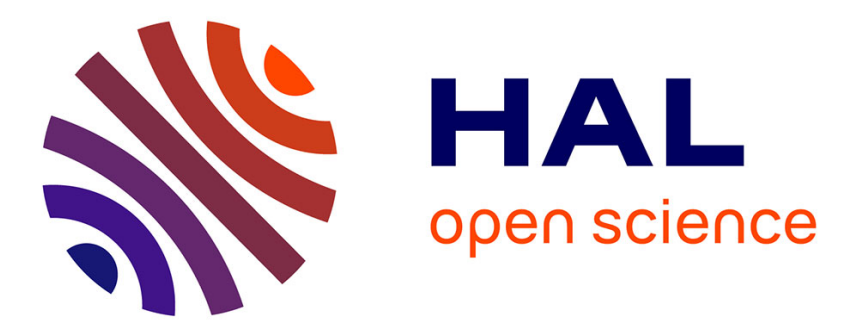

\title{
Energy consumption optimization in 5G networks using multilevel beamforming and large scale antenna systems
}

Fatma Ezzahra Salem, Abdoulaye Tall, Zwi Altman, Azeddine Gati

\section{To cite this version:}

Fatma Ezzahra Salem, Abdoulaye Tall, Zwi Altman, Azeddine Gati. Energy consumption optimization in $5 \mathrm{G}$ networks using multilevel beamforming and large scale antenna systems. IEEE Wireless Communications and Networking Conference (WCNC), 2016, Apr 2016, Doha, Qatar. 10.1109/WCNC.2016.7564904 . hal-01327557

\section{HAL Id: hal-01327557 \\ https://hal.science/hal-01327557}

Submitted on 6 Jun 2016

HAL is a multi-disciplinary open access archive for the deposit and dissemination of scientific research documents, whether they are published or not. The documents may come from teaching and research institutions in France or abroad, or from public or private research centers.
L'archive ouverte pluridisciplinaire HAL, est destinée au dépôt et à la diffusion de documents scientifiques de niveau recherche, publiés ou non, émanant des établissements d'enseignement et de recherche français ou étrangers, des laboratoires publics ou privés. 


\title{
Energy consumption optimization in $5 \mathrm{G}$ networks using multilevel beamforming and large scale antenna systems
}

\author{
Fatma Ezzahra Salem ${ }^{\dagger *}$, Abdoulaye Tall*, Zwi Altman* and Azeddine Gati* \\ ${ }^{\dagger}$ CentraleSupélec, 3 rue Joliot-Curie, 91192 Gif-sur-Yvette, France \\ * Orange Labs, 38/40 rue du Général Leclerc, 92794 Issy-les-Moulineaux, France \\ Email: \{fatma.salem,abdoulaye.tall,zwi.altman,azeddine.gati\}@ orange.com
}

\begin{abstract}
Cellular networks are witnessing an exponential traffic growth leading to an increase in Energy Consumption (EC), and having both environmental and economic impact. Recently, different approaches have been studied to build Green cellular networks focusing mainly on the Base Stations (BSs) as the access network represents $80 \%$ of the total wireless network consumption [1] [2]. One of the promising solutions for increasing throughput and reducing $\mathrm{EC}$ is the deployment of large antenna arrays, known as Large Scale Antenna Systems (LSAS) [3] [4] [5] [6], that can transmit highly focused beams. In the present work, we focus on the use of an advanced BS power model developed within the GreenTouch project [7] [8]. This model allows to quantify the power consumption of a reference scenario comprising multiple sites with standard BS antennas, and then compare it to a LSAS solution implementing multilevel beamforming. We then exploit the LSAS merits to get greener deployment with less BSs, some of which can be turned off as a function of the traffic demand. The coverage areas of each cell can be modified, by updating the codebook of beams. We finally investigate different network configurations which represent distinct trade-offs between EC and capacity. We propose a methodology to represent and design green policies for managing the network which select the desired operating points. Detailed simulation results illustrate the proposed methodology.
\end{abstract}

Keywords-Energy consumption, Green cellular networks, base station power model, multilevel codebook, Green policy management

\section{INTRODUCTION}

Nowadays, cellular networks are facing a tremendous increase in traffic due to the multiplicity of mobile devices and services. Obviously, this growth is accompanied by a high increase in the EC which has a major impact on the overall operational expenditures (OPEX) of the network operators and leads to higher $\mathrm{CO}_{2}$ emissions [9].

The increasing energy costs, the carbon footprint and the concerns for environment led to a new research direction, called Green Radio, aiming at finding solutions towards high Energy-Efficient Networks. Within this direction, several workshops and projects have been organized, for instance CelticOperaNet, FP7-Earth and more recently GreenTouch. Moreover, many activities have been triggered in standardization and regulatory bodies such as 3GPP, ITU, ETSI and ATIS [10] and were dedicated to greening the mobile communications.

The purpose of this study is to contribute to this emerging issue by designing, and, evaluating some advanced solutions focusing mainly on the access network as it consumes most of the energy of the network. In this work, we make use of an advanced power model developed by Interuniversity MicroElectronics Centre (IMEC) [11] [12] [13] [14] within the GreenTouch project that allows to evaluate the BS power consumption. The IMEC model will serve to evaluate the performance of a reference scenario comprising BSs with standard antennas, and compare it to that of a recently proposed technology, namely Multilevel Beamforming which implements a multilevel codebook of beams [15]. The multilevel codebook is constructed according to coverage requirements that enables to select the adequate focused beam for each user. The merits of the proposed beamforming approach are the following: The received signal is amplified by the high gain of the beams, specially the beams of the highest levels; The focused beams create very low interference on neighboring cells, resulting in much higher Spectral Efficiency (SE) and Energy Efficiency (EE). The proposed approach also benefits from low complexity and hence potentially low cost.

The SE is defined as the system throughput per unit of bandwidth. It has always been an efficient criterion enabling to evaluate the performance of wireless networks. The EE has become of major interest for communication systems. However, the two metrics do not progress in the same direction (as shown in Figure 1). As a result a clear trade-off should be reached in order to satisfy the network operators needs. The problem of representing and devising green policies to manage the network is of particular importance and is addressed in the paper.

The contributions of the work can be summarized as follows:

- Exploit the technology evolution in Large Scale Antenna Systems (LSAS) to build more energy-efficient networks.

- Design a LSAS solution with a multilevel codebook according to coverage requirements.

- Analyze the EC of a network implementing the LSAS.

- Design green policies for selecting desired network operation trade-offs between capacity and EC. 


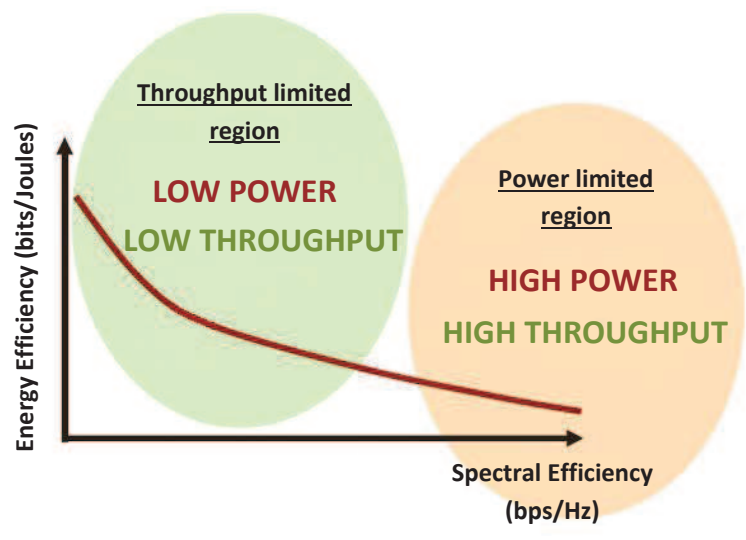

Fig. 1: Trade-off between Energy Efficiency and Spectral Efficiency.

The rest of this paper is organized as follows: Section II presents the multilevel beamforming technology and the power model used in this work. Section III describes the two scenarios considered in this paper as well as a policy design framework for making the network more energy efficient. The performance results obtained from an event-based Long Term Evolution (LTE) network simulator are presented in Section IV and Section V concludes the paper.

\section{SYSTEM MODEL}

This Section presents the system model including the beamforming model using the multilevel codebook and the corresponding beam selection algorithm; the radio modeling and the EC calculation.

\section{A. Multilevel beamforming}

In order to reduce the complexity of beamforming implementation with LSAS, multilevel beamforming has been proposed in [15]. It consists in iteratively focusing the beam towards a given location by navigating through a beam codebook designed in a multilevel manner. Figure 2 represents the coverage maps of the beams at each level of the codebook for one sector. The LSAS comprises a two dimensional array of $\mathrm{Nx} \times \mathrm{Nz}$ equi-spaced radiating dipoles in the $\mathrm{x}$ (horizontal) and $z$ (vertical) directions of the array. The antenna model is described in [15].

The best beam is selected in an iterative manner: for each user $u$, we navigate in the multilevel codebook starting from the lowest level (the full cell) to the narrowest beam. At each step, the beam that maximizes the Signal to Interference plus Noise Ratio (SINR) of the user $u$ among the best beams obtained so far is selected. The algorithm stops when the best beam does not change or the narrowest beams' level is reached. This can be represented as the following optimization problem:

$$
B^{*}=\underset{B \in \text { Codebook }}{\operatorname{argmax}} \operatorname{SINR} R_{u}(B)
$$

where $\operatorname{SINR}_{u}(B)$ is the SINR of user $u$ if he is served by the beam $B$.

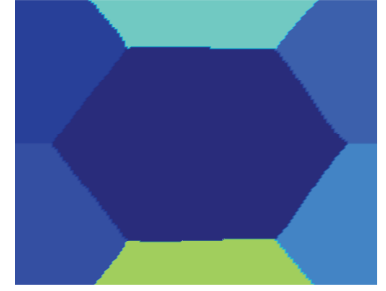

(a) Original Cell

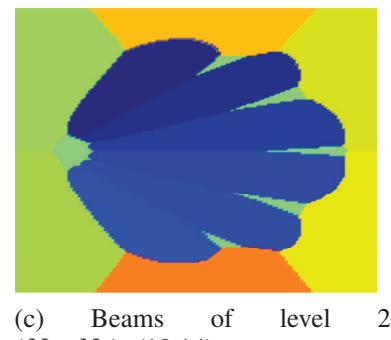

$\left(N_{x}, N_{z}\right)=(10,14)$
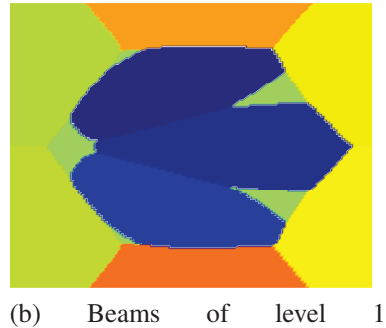
$\left(N_{x}, N_{z}\right)=(5,14)$

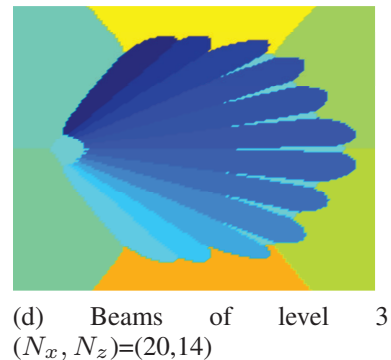

Fig. 2: Multilevel beam coverage maps.

\section{B. Data rate model}

We consider the downlink in this paper. The data rate for a given user $i$ located in a sector $s^{*}$ at time slot $t$ is computed according to the Shannon formula:

$$
R_{i, t}=\alpha_{i, t} W \log \left(1+\operatorname{SINR}_{i, t}\right)
$$

where $W$ represents the frequency bandwidth, $\alpha_{i, t}$ - the fraction of the bandwidth allocated to user $i$ in time slot $t$ and $\operatorname{SINR}_{i, t}$ - the SINR of a user $i$ during a time slot $t$.

$\alpha_{i, t}$ is determined by the scheduling algorithm used and the configuration of users served by sector $s^{*}$. We focus on Round-Robin scheduling thus

$$
\alpha_{i, t}=\frac{1}{n_{s^{*}, t}}
$$

where $n_{s, t}$ is the number of users served by sector $s^{*}$ in time slot $t$.

The SINR is computed as follows:

$$
\operatorname{SINR}_{i, t}=\frac{P_{s^{*}} h_{s^{*}, i}(i)}{I_{i, t}+N_{0}}
$$

where :

- $P_{s^{*}}$ is the transmit power of sector $s^{*}$.

- $h_{s^{*}, l}(k)$ is the channel plus antenna gain from sector $s^{*}$ to user $l$ with the antenna of sector $s^{*}$ configured for user $k$. The antenna configuration mainly concerns the beam used.

- $N_{0}$ is the Noise variance. 
- $I_{i, t}$ is the interference computed as follows

$$
I_{i, t}=\sum_{s \neq s^{*}} \frac{1}{n_{s, t}} \sum_{j \in s} P_{s} h_{s, i}(j)
$$

Remark: The expression for the interference corresponds to an approximation that we consider in order to simplify the computation. Indeed, the exact computation in the simulator would require to know for each BS which users are scheduled in all the other BSs (which beams are used) at each scheduling time of a millisecond. Numerical simulations have shown that the proposed approximation is tight.

\section{Energy Consumption model}

The EC for a single BS $s$ is computed as follows:

$$
E_{k}=\sum_{k=1}^{N_{e}} \tau_{k} P_{s, k}\left(\rho_{s, \tau_{k}}, T_{s}, N_{a n t}\right)
$$

where $N_{e}$ is the number of events, $\tau_{k}$ - the duration between two events $k$ and $k-1$ and $P_{s, k}$ - the power consumption of the BS $s$ during $\tau_{k}$. An event is defined by an arrival or a departure of a user in the network.

$P_{s, k}$ is computed using the IMEC power model tool and it depends on $\rho_{s, \tau_{k}}$ which is the load of the BS $s$ during the period $\tau_{k}$ (we assume data traffic where a scheduled user utilizes all the BS resources. So if BS $s$ serves at least one user during this period, then $\rho_{s, \tau_{k}}=1$, otherwise $\rho_{s, \tau_{k}}=0$ ).

$P_{s, k}$ depends also on the type of the BS which we denote by $T_{s}$, and the number of radiating dipoles in the LSAS, $N_{\text {ant }}$. For a traditional BS with 2x2 MIMO, we have $N_{\text {ant }}=2$ and for a LSAS BS, $N_{\text {ant }}$ depends on the beamforming level used (see Table I).

TABLE I: Number of antennas in the different beamforming levels

\begin{tabular}{|c|c|}
\hline Beamforming level & $\mathbf{N x} \times \mathbf{~ N z}=\mathbf{N}_{\text {ant }}$ \\
\hline Level 0 & 28 \\
\hline Level 1 & $5 \times 14=70$ \\
\hline Level 2 & $10 \times 14=140$ \\
\hline Level 3 & $20 \times 14=280$ \\
\hline
\end{tabular}

For a given BS $s$, there can be more than one user scheduled for transmission during the period $\tau_{k}$ and each user may use a different beamforming level. As a result, $P_{s, k}$ must combine the power consumption of the different scheduled users as follows

$$
P_{s, k}=\sum_{j=1}^{n_{s, k}} \omega_{j, s, k} p_{j, s, k}
$$

where:

- $n_{s, k}$ is the number of users served by the BS $s$ during $\tau_{k}$.

- $\omega_{j, s, k}$ is the fraction of time during which the user $j$ is served by the BS $s$. As we are considering a Round Robin scheduling, $\omega_{j}=\frac{1}{n_{s, k}}$.
- $p_{j, s, k}$ is the power consumption of the user $j$ during period $k$ in which he was served by the BS $s$. It takes into account which beam is used by user $j$ so that we can deduce exactly his power consumption value according to the IMEC tool.

Figure 3 presents the values of $P_{s, k}$ for different configurations: traditional BS denoted as macro, LSAS BS with increasing beamforming levels denoted as Level 0 through Level 3 and with $n_{s, k}=0(\mathrm{OFF})$ and $n_{s, k} \geq 1(\mathrm{ON})$.

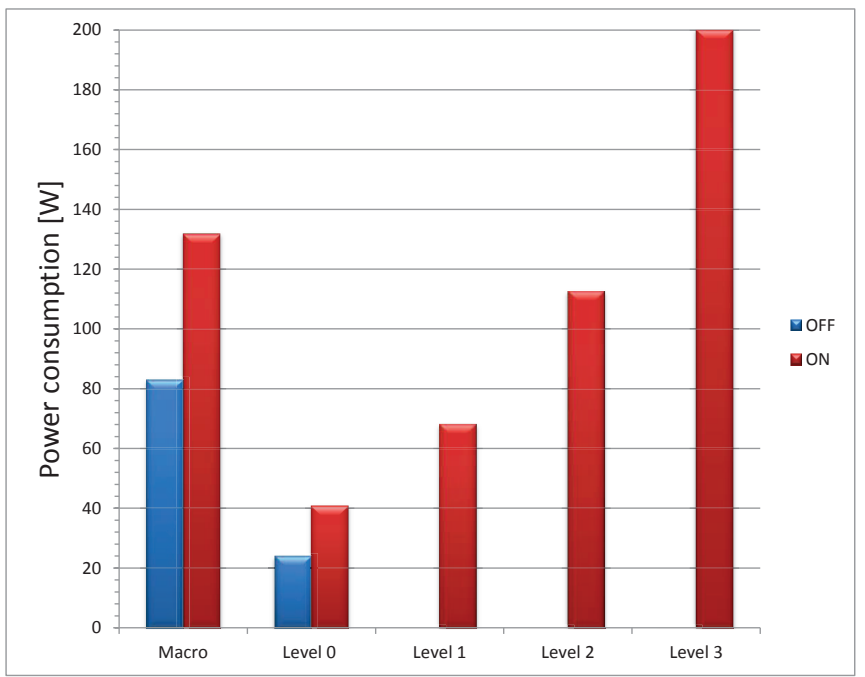

Fig. 3: Power consumption values (Watt). The terms $\mathrm{ON}$ and OFF stand respectively for the cases with and without traffic in the cell.

The total EC of the whole network is the sum of the ECs of all the powered BSs

$$
E_{\text {Total }}=\sum_{k=1}^{N_{B S}} E_{k}
$$

where $N_{B S}$ is the number of BSs.

\section{GREEN NETWORK MODEL}

In order to reduce EC, we study two scenarios making use of the multilevel beamforming technique. We start with a dense hexagonal network of traditional BSs which serves as the reference scenario. FTP type elastic traffic is assumed. The coverage map of the reference scenario is presented in Figure 4.

The first scenario denoted as green scenario consists in switching off one tier of BSs (i.e. of sites) and then using multilevel beamforming to cover the areas previously occupied by the switched off BSs. The coverage map for the level 0 of beams in the multilevel codebook for this scenario is given in Figure 5. The objective of this scenario is to preserve energy by switching off some BSs but preserve the Quality of Service (QoS) using multilevel beamforming. 
In order to use multilevel beamforming in the green scenario, a new beam codebook must be designed tailored specifically to the cells in Figure 5 since each cell has now a specific coverage.

The second scenario denoted as capacitive scenario consists in keeping all BSs switched on but replacing the traditional macro BSs with LSAS BSs and using the multilevel beamforming in every cell. The idea here is to significantly improve the users' SINR and thus reduce the resource utilization due to shorter sojourn time. A reduced resource utilization implies a reduced EC since the system transmits less time at full transmission power. The beam codebook presented in Figure 2 is used for the capacitive scenario.

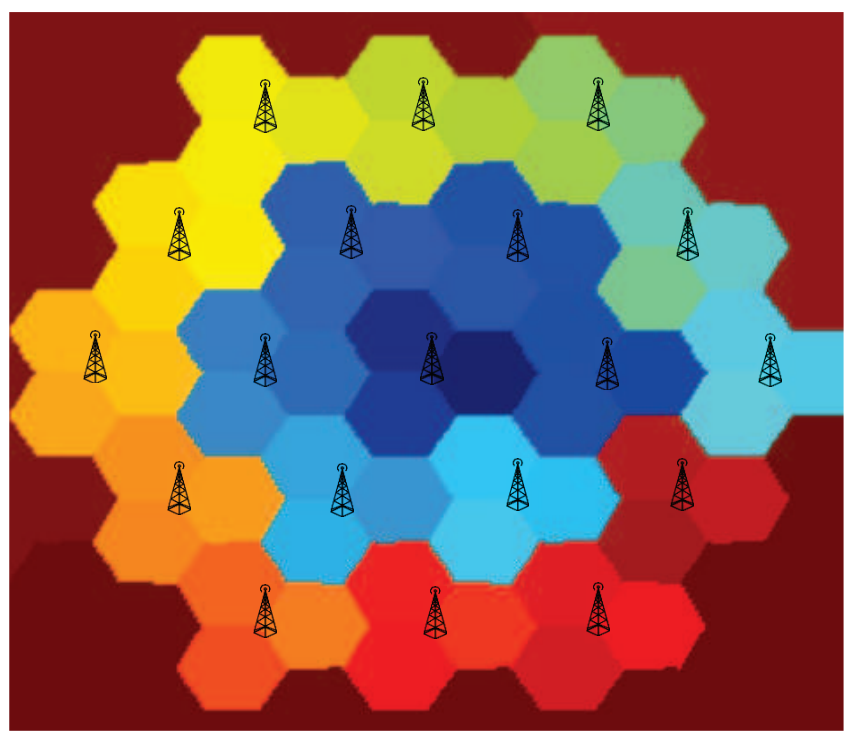

Fig. 4: Hexagonal network deployment.

Other scenarios can be devised in order to exploit the multilevel beamforming technology and each has its advantages and drawbacks. They will perform differently in terms energy efficiency or spectral efficiency as a function of the traffic demand. We propose a framework for comparing and selecting the best scenario according to a green policy that defines a trade-off between energy efficiency and spectral efficiency. Let $S$ be a given scenario, we define a utility function as :

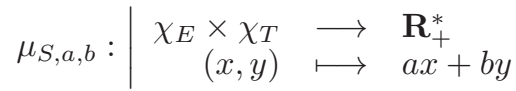

where :

- $\chi_{E}$ and $\chi_{T}$ are, respectively, the sets of energy reduction factors (defined as the ratio between the EC of the reference scenario and that of the considered scenario, capacitive or green), and mean user throughput improvement factors (defined as the ratio between the throughput of the considered scenario and the reference one).

- $a$ and $b$ are, respectively, the weights attributed to each factor that defines the green policy.

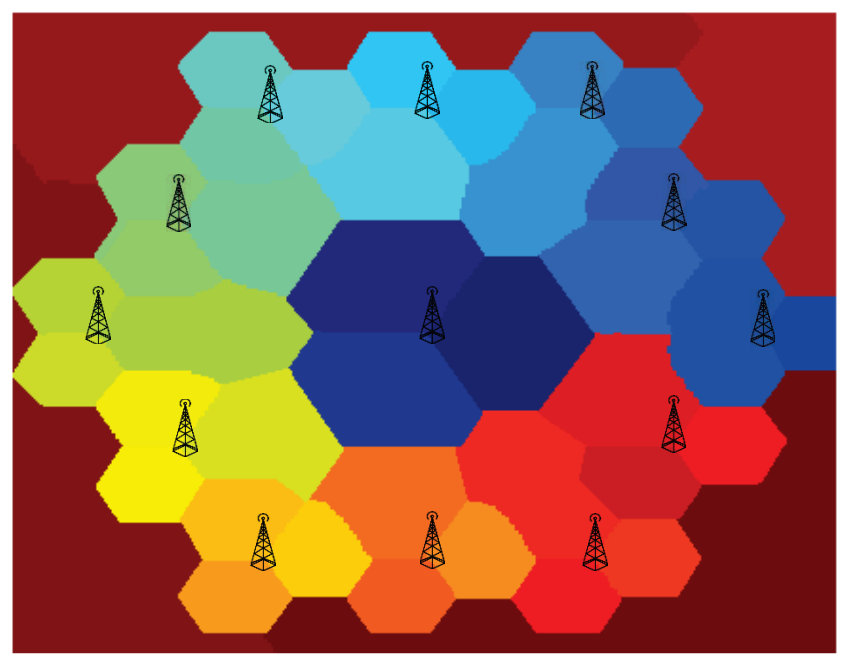

Fig. 5: Coverage map after switching off the middle ring.

The best scenario is chosen as the maximizer of the utility function. The two scenarios we consider represent two policies for operating the network, $S_{1}$ and $S_{2}$. If $\mu_{S_{1}, a, b}\left(x_{1}, y_{1}\right) \geq$ $\mu_{S_{2}, a, b}\left(x_{2}, y_{2}\right)$ then $S_{1}$ is to be deployed and vice versa. The network operator applies its policy by adjusting the weights $a$ and $b$.

\section{NUMERICAL RESULTS}

\section{A. Simulation setup}

We use a LTE event-based simulator coded in Matlab. An event corresponds to an arrival or a departure in the whole network. We assume that the users arrive according to a Poisson process, download a file of exponential size with mean of 4 Mbits, and leave the network as soon as their download is complete. No mobility is considered in the simulations but the users arrive at random locations in the whole network. We focus on a dense urban case with an inter-site distance of 500 $\mathrm{m}$.

In the reference scenario, we consider a hexagonal network of 19 tri-sectorized conventional macro BSs (see Figure 4) with $2 \times 2$ MIMO antennas, antenna heights of $30 \mathrm{~m}$ and maximum antenna gains of $18 \mathrm{dBi}$. In the capacitive and green scenarios, we replace the macro BSs of the reference scenario with LSAS BSs in order to perform multilevel beamforming.

The simulation parameters are summarized in Table II.

\section{B. Performance results}

We consider four performance indicators to compare the different scenarios: Mean User Throughput (MUT), Cell-Edge Throughput (CET), load and EC. Table III presents the performance results for each scenario with varying arrival rates.

The performance results show that multilevel beamforming is a promising solution for increasing the throughput and decreasing the EC of the network. The user throughput improvement comes from the increase of the antenna gain 
TABLE II: Simulator characteristics

\begin{tabular}{|c|c|}
\hline \multicolumn{2}{|c|}{ Network parameters } \\
\hline Macro Cell layout & hexagonal trisector \\
\hline Antenna height & $30 \mathrm{~m}$ \\
\hline Bandwidth & $10 \mathrm{MHz}$ \\
\hline Scheduling type & Round Robin \\
\hline \multicolumn{2}{|c|}{ Channel characteristics } \\
\hline Thermal noise & $-174 \mathrm{dBm} / \mathrm{Hz}$ \\
\hline Path loss $(d$ in km) & $128.1+37.6 \log _{10}(d) \mathrm{dB}$ \\
\hline Shadowing & Log-normal $(6 \mathrm{~dB})$ \\
\hline \multicolumn{2}{|c|}{ Traffic characteristics } \\
\hline Service type & FTP \\
\hline Average file size & $4 \mathrm{Mbits}$ \\
\hline
\end{tabular}

TABLE III: Simulation results

\begin{tabular}{|c|c|c|c|c|c|}
\hline $\begin{array}{c}\text { Arrival rate } \\
{\left[\text { users/s/Km } \mathbf{K m}^{2}\right]}\end{array}$ & Scenario & $\begin{array}{c}\mathbf{E C} \\
{[\mathbf{W h}]}\end{array}$ & $\begin{array}{c}\text { MUT } \\
{[\mathbf{M b p s}]}\end{array}$ & $\begin{array}{c}\text { CET } \\
{[\mathbf{M b p s}]}\end{array}$ & $\begin{array}{c}\text { Load } \\
{[\mathbf{\%}]}\end{array}$ \\
\hline \multirow{3}{*}{$\lambda_{1}=3$} & Reference & 135.67 & 26.76 & 6.7 & 4 \\
\cline { 2 - 6 } & Capacitive & 42.92 & $\mathbf{4 0 . 7 4}$ & $\mathbf{1 1 . 8 9}$ & $\mathbf{3}$ \\
\cline { 2 - 6 } & Green & $\mathbf{3 2 . 0 6}$ & 30.67 & 7.84 & 5.5 \\
\hline \multirow{3}{*}{$\lambda_{2}=6$} & Reference & 139.73 & 25 & 5.98 & 9.5 \\
\cline { 2 - 6 } & Capacitive & 48.43 & $\mathbf{3 9 . 7}$ & $\mathbf{1 1 . 6}$ & $\mathbf{6}$ \\
\cline { 2 - 6 } & Green & $\mathbf{3 9 . 4 8}$ & 29.6 & 5.8 & 11.7 \\
\hline \multirow{3}{*}{$\lambda_{3}=10$} & Reference & 146.17 & 21.9 & 4.6 & 18 \\
\cline { 2 - 6 } & Capacitive & 53.94 & $\mathbf{3 8 . 8 6}$ & $\mathbf{9 . 9 8}$ & $\mathbf{9}$ \\
\cline { 2 - 6 }$\lambda_{4}=13$ & Green & $\mathbf{4 8 . 4 9}$ & 26.23 & 3.8 & 20 \\
\hline \multirow{3}{*}{$\lambda_{5}=15$} & Reference & 151.66 & 19.7 & 4.08 & 25 \\
\cline { 2 - 6 } & Capacitive & 59.5 & $\mathbf{3 6 . 7}$ & $\mathbf{8 . 1}$ & $\mathbf{1 3}$ \\
\cline { 2 - 6 } & Green & $\mathbf{5 7 . 1 1}$ & 24.14 & 2.7 & 30 \\
\hline & Reference & 154.4 & 18.68 & 3.79 & 28.7 \\
\cline { 2 - 6 } & Capacitive & $\mathbf{6 2 . 1 7}$ & $\mathbf{3 5 . 9}$ & $\mathbf{7 . 2}$ & $\mathbf{1 5}$ \\
\cline { 2 - 6 } & Green & 65.78 & 21.16 & 1.3 & 35 \\
\hline \multirow{3}{*}{$\lambda_{6}=20$} & Reference & 166.65 & 14.18 & 2.15 & 45 \\
\cline { 2 - 6 } & Capacitive & $\mathbf{7 0 . 1 4}$ & $\mathbf{3 3 . 9}$ & $\mathbf{5 . 7}$ & $\mathbf{2 2}$ \\
\cline { 2 - 6 } & Green & 81.64 & 14.8 & 0.24 & 49 \\
\hline
\end{tabular}

obtained by using a large antenna array. The sojourn time of the users in the network is reduced resulting in lower load values in the capacitive scenario as shown in Table III. It is noted that the load value is higher in the green scenario compared to the reference scenario. This can be explained by the fact that we shut down some cells so each of the remaining ones will serve more users.

The EC reduction of the capacitive scenario with respect to the reference one is mainly due to the shorter mean sojourn time of the former. In the green scenario, the reduction of the $\mathrm{EC}$ is the result of the decrease in the number of the active BSs.

The EC reduction in both capacitive and green scenarios is also linked to the transmission schemes in terms of the beam level selection probabilities.

Multilevel beamforming is clearly an efficient solution, both for improving energy and throughput. However, the capacitive scenario can become greener (with lower energy consumption) than the green scenario in high load scenarios. The best scenario must then be selected and deployed according to the traffic demand.

The energy and spectral efficiency of the capacitive and the green scenarios depend on the traffic demand. For very high loads ( $\lambda \geq 15$ in Table III), the capacitive scenario is both more spectrally efficient and more energy efficient than the green scenario. This is due to the fact that in multilevel beamforming, the most used beams to serve users are in the highest level (see Figure 6) which consumes 8 times more energy than the level 0 beams (see Figure 3 ) which are used during idle times. Thus, the EC will highly depend on the load of the system. In high traffic demand scenarios, the load of the capacitive scenario is much lower than in the green scenario, thus the capacitive scenario uses less often the highest levels of beams leading to a lower EC.

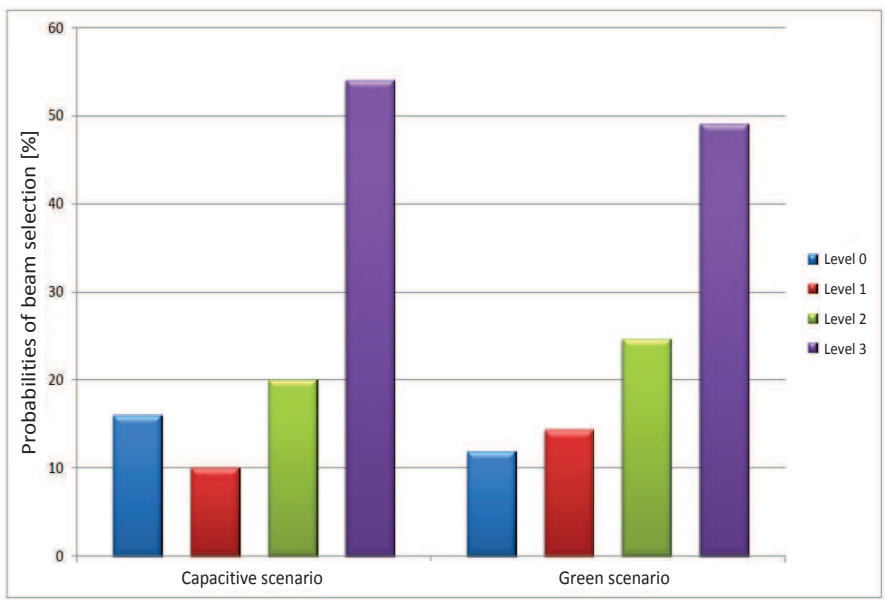

Fig. 6: Probabilities of selection of the different beam levels in the Capacitive and the Green scenarios (for $\lambda_{6}$ as an example).

\section{Energy-Efficient Network management}

Figure 7 presents the performance of the green and capacitive scenarios in the EC-MUT plane. Zone one (on the left of the dashed red line) corresponds to the zone where it can be beneficial to shift from one scenario to the other. This shift corresponds to the trade-off between the EC reduction and the user throughput improvement. The choice of the scenario depends on the operator policy, namely on the relative importance given to each criterion.

Zone 2 corresponds to the values of $\lambda$ where the capacitive scenario is better both for EC and MUT. The red dashed line sets the limit between Zones 1 and 2 . It corresponds to the arrival rate $\lambda_{4}$ at which at least one BS in the Green Scenario has a load equal to $80 \%$, and starting from which the green scenario is no longer interesting.

We apply the green network policy framework discussed in Section III. Three different policies are considered with 
corresponding parameters specified in Table IV. The best policy performance is highlighted in bold. The results for applying these policies are also shown in Figure 7.

TABLE IV: Utility values for the two scenarios

\begin{tabular}{|c|c|c|c|}
\hline Policies & Arrival rate & $\mu_{\text {capacitive }, a, b}$ & $\mu_{\text {green }, a, b}$ \\
\hline \hline \multirow{2}{*}{$\begin{array}{c}\text { Policy 1 } \\
(\mathbf{a}, \mathbf{b})=\mathbf{( 1 , 1 )}\end{array}$} & $\lambda_{1}$ & 4.68 & $\mathbf{5 . 3 8}$ \\
\cline { 2 - 4 } & $\lambda_{2}$ & 4.47 & $\mathbf{4 . 7 2}$ \\
\cline { 2 - 4 } & $\lambda_{3}$ & $\mathbf{4 . 4 8}$ & 4.2 \\
\hline \hline \multirow{2}{*}{$\begin{array}{c}\text { Policy 2 } \\
(\mathbf{a}, \mathbf{b})=(\mathbf{0 . 3}, \mathbf{0 . 7})\end{array}$} & $\lambda_{1}$ & 2.67 & $\mathbf{3 . 3 1}$ \\
\cline { 2 - 4 } & $\lambda_{2}$ & 2.5 & $\mathbf{2 . 8 3}$ \\
\cline { 2 - 4 } & $\lambda_{3}$ & 2.43 & $\mathbf{2 . 4 7}$ \\
\hline \hline \multirow{2}{*}{$\begin{array}{c}\text { Policy 3 } \\
(\mathbf{a}, \mathbf{b})=(\mathbf{0 . 7}, \mathbf{0 . 3})\end{array}$} & $\lambda_{1}$ & 2.01 & $\mathbf{2 . 0 7}$ \\
\cline { 2 - 4 } & $\lambda_{2}$ & $\mathbf{1 . 9 8}$ & 1.89 \\
\cline { 2 - 4 } & $\lambda_{3}$ & $\mathbf{2 . 0 6}$ & 1.74 \\
\hline
\end{tabular}

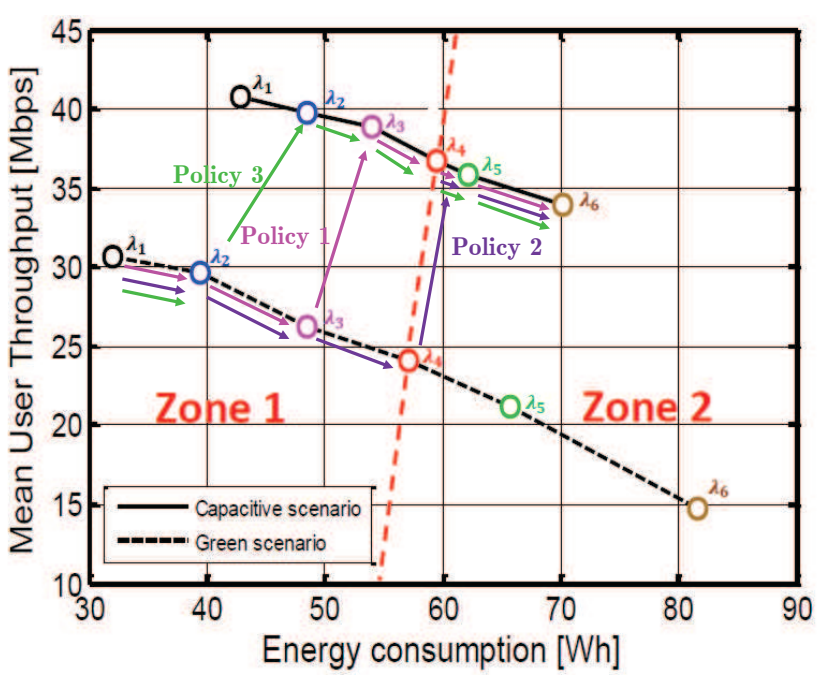

Fig. 7: Illustration of the different deployment policies.

For a given policy, the utility can be monitored, and the shift between the two scenarios occurs when one utility gets better than the other. Alternatively, one can compute offline a table which maps arrival rates onto utility values and identify the switching points from one scenario to the other.

\section{CONCLUSion}

We have presented in this paper two scenarios in which multilevel beamforming has been used to decrease EC either by switching off some BSs or by increasing the system capacity thus reducing its load. We also presented a framework for designing a green policy that allows to switch between different scenarios according to the network operator objectives. This framework implements a trade-off between energy efficiency and spectral efficiency. More scenarios can be devised for leveraging the potential of multilevel beamforming in making mobile networks more energy efficient such as reducing the transmission power when using beamforming or load balancing using adequately designed codebooks. It is noted that the performance results obtained for the energy consumption are highly dependent on the power model used, i.e. here the IMEC power model. Other power models can be considered and the performance of the scenarios proposed can be evaluated with these models. This paper has the ambition to foster research on green wireless networks management using self-organized features. The paper has shown that a trade-off has to be found between SE and EE. Moreover, different scenarios have to be studied e.g. rural, in order to evaluate their operability.

\section{REFERENCES}

[1] Nokia Siemens Networks, "ETSI RRS05-024,” 2011.

[2] University of Melbourne, "The power of wireless cloud," White Paper of Centre for Energy-Efficicent Telecommunications (CEET), 2013.

[3] T. Marzetta, "Noncooperative cellular wireless with unlimited numbers of base station antennas," IEEE Transactions on Wireless Communications, Nov 2010.

[4] F. Rusek, D. Persson, B. K. Lau, E. Larsson, T. Marzetta, O. Edfors, and F. Tufvesson, "Scaling Up MIMO: Opportunities and Challenges with Very Large Arrays,” IEEE Signal Processing Magazine, Jan 2013.

[5] E. Larsson, O. Edfors, F. Tufvesson, and T. Marzetta, "Massive MIMO for next generation wireless systems," IEEE Communications Magazine, Feb 2014.

[6] E. Bjornson, L. Sanguinetti, J. Hoydis, and M. Debbah, "Designing multi-user MIMO for energy efficiency: When is massive MIMO the answer?" in IEEE Wireless Communications and Networking Conference (WCNC), April 2014.

[7] GreenTouch, http://greentouch.org/, accessed: 2016-09-29.

[8] GreenTouch white paper, "GreenTouch green meter research study: Reducing the net energy consumption in communications networks by up to $90 \%$ by 2020 ," June 2013. [Online]. Available: www.greentouch.org/uploads/documents/GreenTouch_Green_Meter_Research_Study_26_June_2013.pdf

[9] R. Litjens, Y. Toh, H. Zhang, and O. Blume, "Assessment of the energy efficiency enhancement of future mobile networks," in IEEE Wireless Communications and Networking Conference (WCNC), April 2014.

[10] L. Correia, D. Zeller, O. Blume, D. Ferling, Y. Jading, I. Godor, G. Auer, and L. Van der Perre, "Challenges and enabling technologies for energy aware mobile radio networks," IEEE Communications Magazine, Nov 2010 .

[11] Interuniversity Microelectronics Centre (IMEC) website, http://www.imec.be/, accessed: 2015-09-29.

[12] C. Desset, B. Debaillie, and F. Louagie, "Towards a flexible and futureproof power model for cellular base stations," in 24th Tyrrhenian International Workshop on Digital Communications - Green ICT (TIWDC), Sept 2013.

[13] _ - "Modeling the hardware power consumption of large scale antenna systems," in Green Communications (OnlineGreencomm), 2014 IEEE Online Conference on, Nov 2014.

[14] B. Debaillie, C. Desset, and F. Louagie, "A flexible and futureproof power model for cellular base stations," in IEEE 81st Vehicular Technology Conference (VTC Spring), May 2015.

[15] A. Tall, Z. Altman, and E. Altman, "Multilevel beamforming for high data rate communication in 5G networks," arXiv preprint arXiv:1504.00280, 2015. 